Draft Version May 29, 2018

Typeset using LATEX twocolumn style in AASTeX62

\title{
Kinematic evidence for an embedded protoplanet in a circumstellar disc
}

\author{
C. Pinte, ${ }^{1,2}$ D. J. Price, ${ }^{1}$ F. Ménard ${ }^{2}$ G. Duchêne,${ }^{3,2}$ W. R. F. Dent,${ }^{4}$ T. Hill,${ }^{4}$ I. De Gregorio-Monsalvo, ${ }^{4}$ \\ A. Hales, ${ }^{4,5}$ AND D. Mentiplay ${ }^{1}$ \\ ${ }^{1}$ Monash Centre for Astrophysics (MoCA) and School of Physics and Astronomy, Monash University, Clayton Vic 3800, Australia \\ ${ }^{2}$ Univ. Grenoble Alpes, CNRS, IPAG, F-38000 Grenoble, France \\ ${ }^{3}$ Astronomy Department, University of California, Berkeley, CA 94720-3411, USA \\ ${ }^{4}$ Atacama Large Millimeter / Submillimeter Array, Joint ALMA Observatory, Alonso de Córdova 3107, Vitacura 763-0355, Santiago, \\ Chile \\ ${ }^{5}$ National Radio Astronomy Observatory, 520 Edgemont Road, Charlottesville, VA 22903-2475, United States of America
}

\begin{abstract}
Discs of gas and dust surrounding young stars are the birthplace of planets. However, the direct detection of protoplanets forming within discs has proved elusive to date. We present the detection of a large, localized deviation from Keplerian velocity in the protoplanetary disc surrounding the young star HD 163296. The observed velocity pattern is consistent with the dynamical effect of a two-Jupiter-mass planet orbiting at a radius $\approx 260$ au from the star.
\end{abstract}

Keywords: stars: individual (HD 163296) — protoplanetary discs — planet-disc interaction — submillimeter: planetary systems — hydrodynamics — radiative transfer

\section{INTRODUCTION}

Direct observations of forming planets in protoplanetary discs is the ultimate goal of disc studies. The disc usually outshines the planet, requiring observations at high contrast and angular resolution. Detections by direct imaging have been reported in several discs: HD 100546 (Quanz et al. 2013a; Brittain et al. 2014; Quanz et al. 2015; Currie et al. 2015), LkCa 15 (Kraus \& Ireland 2012; Sallum et al. 2015), HD 169142 (Quanz et al. 2013b; Biller et al. 2014; Reggiani et al. 2014), and MWC 758 (Reggiani et al. 2018). Yet, most of the detections to date have been subsequently challenged (e.g., Thalmann et al. 2015, 2016; Rameau et al. 2017; Ligi et al. 2018). The quest continues.

An alternative approach is to search for indirect signatures imprinted by planets on their host disc. The Atacama Large Millimetre Array (ALMA), and adaptive optics systems have revealed a variety of structures: gaps and rings (ALMA Partnership et al. 2015; Andrews et al. 2016; Isella et al. 2016), spirals (e.g. Benisty et al. 2015; Stolker et al. 2016), that could be signposts of planets, but numerous other explanations also exist that do not require planets (e.g. Takahashi \& Inutsuka 2014; Flock et al. 2015; Gonzalez et al. 2015; Lorén-Aguilar \& Bate

christophe.pinte@monash.edu
2015; Zhang et al. 2015; Béthune et al. 2016). Embedded planets in circumstellar discs will launch spiral waves at Lindblad resonances both inside and outside of their orbit (e.g. Ogilvie \& Lubow 2002), disturbing the local Keplerian velocity pattern. Hydrodynamic simulations show that the impact on the velocity pattern should be detectable by high spectral resolution ALMA line observations (Perez et al. 2015). Deviations from Keplerian rotation have been detected around circumbinary discs, with streamers at near free-fall velocities (Casassus et al. 2015; Price et al. 2018) and radial flows or warps (Walsh et al. 2017).

HD 163296 is a $\sim 4.4 \mathrm{Myr}$ old Herbig Ae star located at a distance of $101.5 \pm 1.2 \mathrm{pc}$ from the Sun (Gaia Collaboration et al. 2018). We rescaled all relevant quantities from previous papers based on the new Gaia distance. HD 163296 has a mass of $1.9 \mathrm{M}_{\odot}$ (e.g. Flaherty et al. 2015), a luminosity of $25 \mathrm{~L}_{\odot}$ (Natta et al. 2004), and an A1Ve spectral type, with effective temperature $9300 \mathrm{~K}$. Observations with the Hubble Space Telescope (HST) revealed a disc in scattered light that extends as far out as 375 au (Grady et al. 2000). Interestingly, Grady et al. (2000) inferred the presence of a giant planet at $\approx 270 \mathrm{au}$ based on the gap observed in scattered light at that radius. de Gregorio-Monsalvo et al. (2013) presented ALMA data and showed that the gaseous component of the disc extends to distances of 


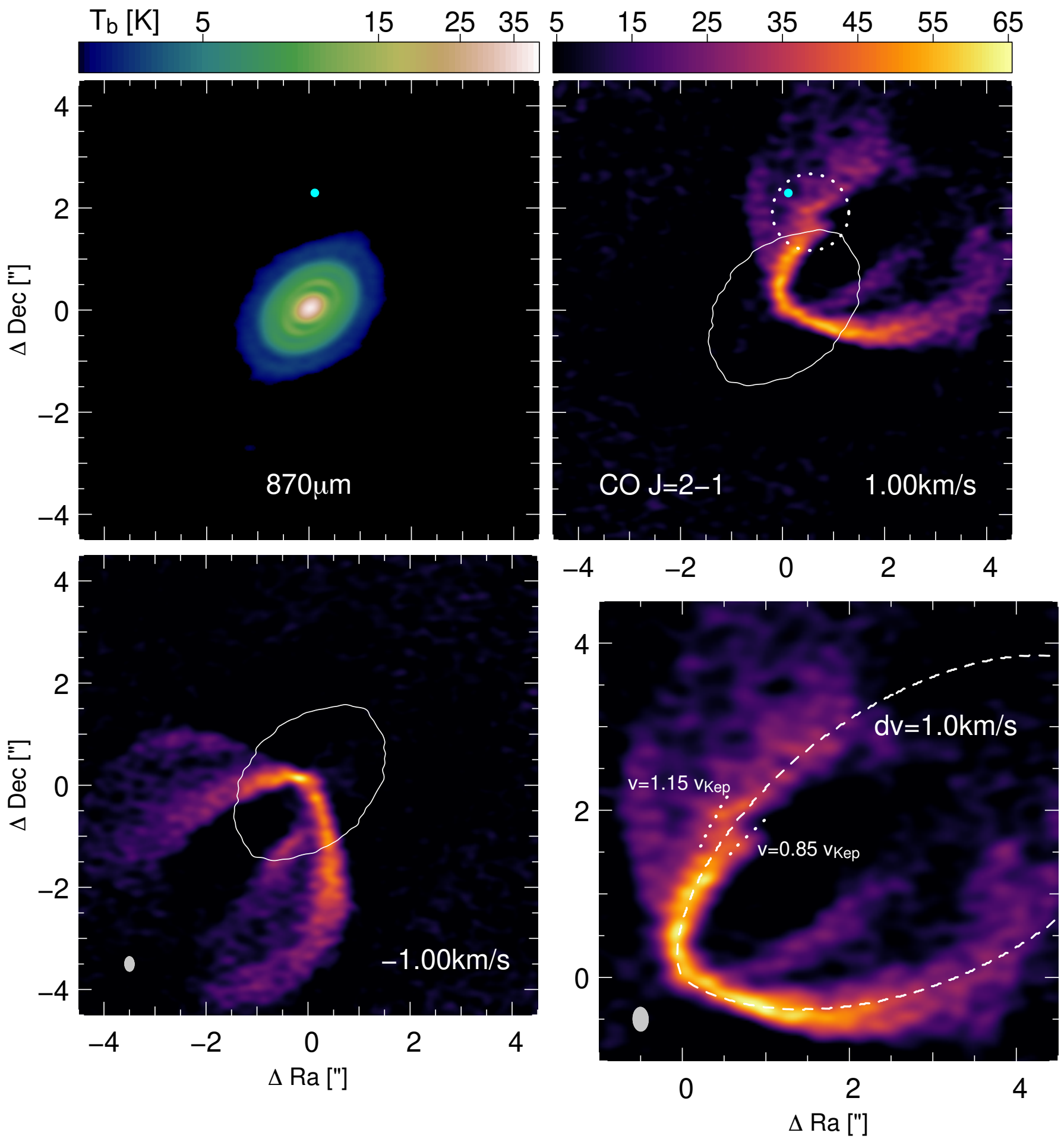

Figure 1. Kinematic asymmetry in HD 163296. Band 6 continuum emission (top left) and channel map of ${ }^{12}$ CO line emission at $+1 \mathrm{~km} \mathrm{~s}^{-1}$ from the systemic velocity (top right, with a close-up shown in bottom right) shows a distinct 'kink' in the emission (highlighted by the dotted circle). Comparison with the continuum emission (top left) locates this outside of the outermost dust ring. The corresponding emission on the opposite side of the disc (bottom left; showing $-1 \mathrm{~km} \mathrm{~s}^{-1} \mathrm{channel}$ ) shows no corresponding feature, indicating the disturbance to the flow is localised in both radius and azimuth. The channel width is $\Delta v=0.1 \mathrm{~km} \mathrm{~s}^{-1}$. The white contour shows the $5-\sigma\left(\sigma=0.1 \mathrm{mJy}^{\mathrm{beam}}{ }^{-1}\right)$ level of the continuum map. The dashed line is the expected location of the isovelocity curve on the upper surface of a disc with an opening angle of $15^{\circ}$ and an inclination of $45^{\circ}$. Dotted lines in the bottom-right figure indicate $15 \%$ deviations $\left(\approx 0.4 \mathrm{~km} \mathrm{~s}^{-1}\right)$ from Keplerian flow around the star. The potential planet location is marked by a cyan dot, assuming it is located in the midplane. 
at least $\mathrm{R}_{\text {out-CO }}=415 \mathrm{au}$ in $\mathrm{CO}$ while the continuum is detected only to $R_{\text {out-Dust }}=200$ au. Higher resolution ALMA imaging revealed a bright inner disc component within the inner $0 !^{\prime \prime} 5$, and a spectacular series of three rings at $\approx 65 \mathrm{au}, 100 \mathrm{au}$, with a fainter ring at $160 \mathrm{au}$ (Isella et al. 2016).

In this Letter, we present the detection of a local deviation from the Keplerian velocity pattern found in high spectral resolution ALMA imaging. By comparing with models we find this to be consistent with the presence of a few-Jupiter mass protoplanet in the disc.

\section{OBSERVATIONS AND DATA REDUCTION}

We use archival ALMA data. Observations were performed on 2012 June 9, 11, 22, and July 6 at Band 7 (2011.0.000010.SV), and on 2015 August 5, 8, and 9 at Band 6 (2013.1.00601.S). A complete description of the data was presented in de Gregorio-Monsalvo et al. (2013) and Isella et al. (2016). For the Band 7 data, we re-used the maps produced by de Gregorio-Monsalvo et al. (2013), with a $0.52^{\prime \prime} \times 0.38^{\prime \prime}$ beam at $\mathrm{PA}=82^{\circ}$, and a channel width of $0.11 \mathrm{~km} \mathrm{~s}^{-1}$.

We used CASA scripts provided by ALMA to calibrate the Band 6 data. As the data from the night of August 9 showed significantly higher noise and flux levels, we selected only the data from the August 5 and 8 for the analysis. We performed three successive rounds of phase self-calibration, the last with solutions calculated for each individual integration (6s), followed by a phase and amplitude self-calibration. The continuum self-calibration solutions were applied to the CO lines. Imaging was performed at $0.1 \mathrm{~km} \mathrm{~s}^{-1}$ resolution, using Briggs weighting with a robust parameter of -0.5 to obtain a synthesized beam of $0.28^{\prime \prime} \times 0.18^{\prime \prime}$ at $\mathrm{PA}=-88^{\circ}$. We did not subtract the continuum emission in order to avoid underestimating the gas temperature and affecting the apparent morphology of the emission (e.g. Weaver et al. 2018). At the location of the detected velocity deviation, continuum emission is negligible, and an analysis on continuum-subtracted data would lead to the same results.

\section{RESULTS AND ANALYSIS}

The disc shows the typical butterfly pattern of discs in Keplerian rotation (de Gregorio-Monsalvo et al. 2013; Rosenfeld et al. 2013). In a given channel, the emission is concentrated along an isovelocity curve, corresponding to the region of the disc where the projected velocity is equal to the channel velocity. The emission from the upper and lower surfaces - above and below the midplane as seen by the observer - and from the near and far sides of these surfaces, is well separated (Fig. 1, and schematic view in Fig. 3).
In a recent paper (Pinte et al. 2018) we showed how to reconstruct the position and velocity of each of the $\mathrm{CO}$ layers, for discs at intermediate inclination, by simple geometrical arguments based on the emission in each channel map. HD 163296 displays a similar scale height and velocity profile to the T Tauri star IM Lupi (Pinte et al. 2018), with a flared CO emitting surface and decreasing velocities and temperature with radius (C. Pinte et al., in prep.).

Significantly, HD 163296 shows an asymmetry between the southeast and northwest sides of the disc at a cylindrical radius of $\approx 260 \mathrm{au}$, outside of the third dust ring seen in continuum emission. This asymmetry is most evident in channels at a projected velocity of $\approx 6.8 \pm 0.2 \mathrm{~km} \mathrm{~s}^{-1}\left(\approx 1 \mathrm{~km} \mathrm{~s}^{-1}\right.$ from the systemic velocity). Fig. 1 shows the corresponding individual velocity channels. The emission feature - highlighted by the dotted circle - corresponds to a kink in the uppersurface isovelocity curve northwest of the central object at velocities close to $\mathrm{d} v=+1 \mathrm{~km} \mathrm{~s}^{-1}$. The symmetric channel $\left(\mathrm{d} v=-1 \mathrm{~km} \mathrm{~s}^{-1}\right.$,) shows a smooth Keplerian profile to the southeast. We detect a similar deformation of the isovelocity curves at the same location in both ${ }^{12} \mathrm{CO} J=2-1$ and $3-2$ transitions (Fig. 2). While it is not as obvious in the Band 7 Early-Science data due to the limited spatial resolution, the deformation of the isovelocity curve is present and could already be seen, with the benefit of hindsight from our Band 6 detection, in de Gregorio-Monsalvo et al. (2013) and Rosenfeld et al. (2013, their Fig. 3 and 2, respectively). The asymmetry is not detectable in the less abundant isotologues ${ }^{13} \mathrm{CO}$ and $\mathrm{C}^{18} \mathrm{O}$, where the emission is more diffuse and fainter because of the lower optical depth.

The deformation of the emission is localised to an area approximately $0.5^{\prime \prime}$ in size (indicated by the dotted circle in Figures 1 and 2) and to channel maps at velocities between 0.8 and $1.2 \mathrm{~km} \mathrm{~s}^{-1}$ from the systemic velocity (top row of Fig. 2). This argues for a localised perturbation and excludes an origin from any large-scale structure in the disc.

\section{MODELS AND DISCUSSION}

The detected asymmetry matches our expectations for a local deviation from Keplerian velocity caused by a massive body embedded in the disc. A local deviation of $\approx 0.4 \mathrm{~km} \mathrm{~s}^{-1}$ is enough to reproduce the observed spatial shift (Fig. 1, bottom-right panel). The dotted lines shown in the bottom-right panel delineate what would be $\approx 15 \%$ deviations in the local velocity field, which is the approximate extent of the deviation from Keplerian rotation. Most significantly, the shape of the deviation in the emission maps is similar to the prediction by Perez 

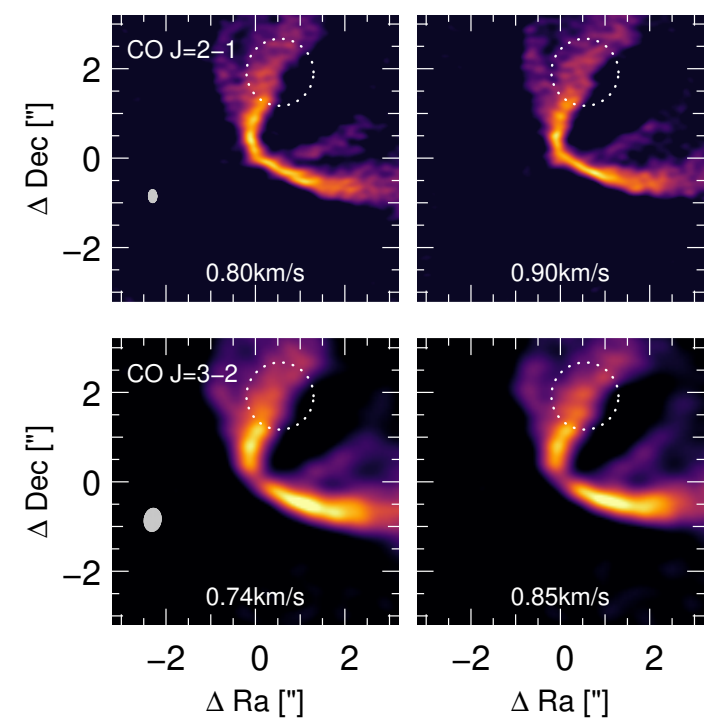
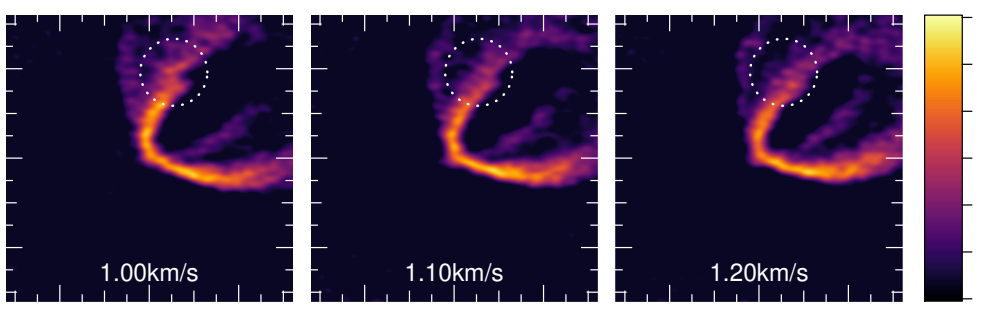

65

55

45

35 을

$25^{\circ}$

15
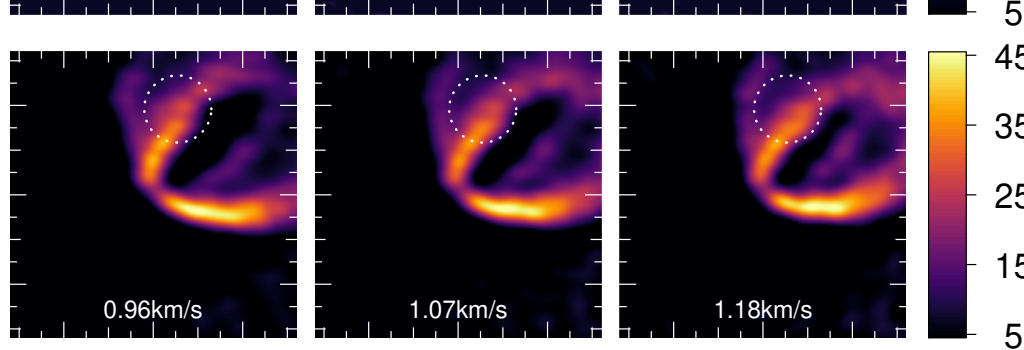

45

35

$25 \frac{\bar{Y}}{\circ}$ 15

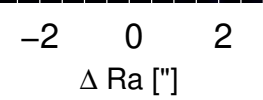

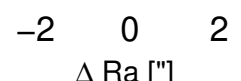

$\Delta \mathrm{Ra}["]$

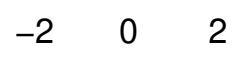

$\Delta \mathrm{Ra}["]$

Figure 2. Channel maps around the detected deviation from Keplerian velocity. The 'kink' is most visible in channels at velocities between 0.8 and $1.2 \mathrm{~km} / \mathrm{s}$ (top row) and is also seen in the $\mathrm{J}=3-2$ transition in similar velocity channels (bottom row) indicating it is localised in both space and velocity.

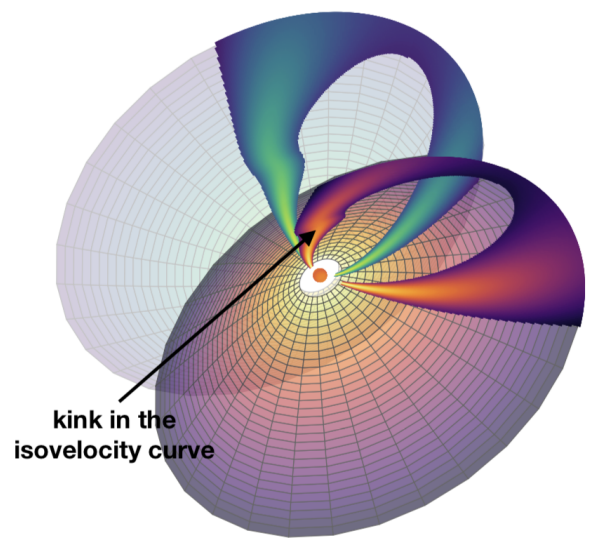

Figure 3. Geometry of the inclined and flared disc, showing a schematic of the expected emission from two infinitely thin emitting surfaces. Green shows the emission from the lower surface of the disc, and red shows the upper surface. We added a $10 \%$ deviation in azimuthal velocity north of the star, which appears as a 'kink' in the line emission. Emission is only seen when the projected velocity matches the channel velocity, producing the characteristic 'butterfly' shape. Emission is preferentially seen on the upper surface of the disc due to the higher inclination with respect to the line of sight.

et al. (2015) for the kinematic signatures of an embedded planet, where the wake of the spiral generated by the planet was shown to produce a kink in the emission due to the deviation from the Keplerian rotation around the central star.

The basic feature of the channel maps can be explained with a simple model assuming emission from two infinitely thin emitting surfaces. Figure 3 shows the ex- pected emission arising from such a model, showing the butterfly signature from the disc. Asymmetries of the velocity field, added in an ad hoc manner in the model for illustrative purposes, are evident as small bumps on the line emissions.

To go beyond this simple model and infer the mass of the putative planet, we performed a series of $3 \mathrm{D}$ global simulations using the PHANTOM Smoothed Particle Hydrodynamics (SPH) code (Price et al. 2017). We adopted the gas disc parameters from de GregorioMonsalvo et al. (2013). We employed gas-only simulations, ignoring the effect of dust, using 1 million $\mathrm{SPH}$ particles and a central mass of $1.9 \mathrm{M}_{\odot}$. The inner radius of the disc in our model was set to 50 au (mainly to speed up the calculations as the inner disc is irrelevant for our present purpose), with an initial outer radius set to $500 \mathrm{au}$. We set the gas mass between those radii to $10^{-2} \mathrm{M}_{\odot}$, and use an exponentially tapered power-law surface density profile with a critical radius of $100 \mathrm{au}$, power-law index of $p=-1.0$, and an exponent $\gamma=0.8$. The disc aspect ratio was set to 0.08 at $50 \mathrm{au}$, with a vertically isothermal profile. We set the artificial viscosity in the code in order to obtain an average Shakura \& Sunyaev (1973) viscosity of $10^{-3}$ (Lodato \& Price 2010), in agreement with the upper limits found by Flaherty et al. (2015, 2017).

We embedded a single planet in the disc orbiting at $260 \mathrm{au}$ with a mass of either $1,2,3$, or $5 \mathrm{M}_{\mathrm{Jup}}$. We used sink particles (Bate et al. 1995) to represent the star and planet. We set the accretion radius of the planet to half of the Hill radius (7.05, 8.85, 10.15 and $12 \mathrm{au}$, respectively), with an accretion radius of $10 \mathrm{au}$ for the 
central star. The model surface density is plotted in Fig. 4 for the $2 \mathrm{M}_{\text {jup }}$ planet. We evolved the models for 35 orbits of the planet $(\approx 100,000$ years $)$, which is sufficient to establish the flow pattern around the planet.

To compute the temperature and synthetic line maps, we used the MCFOST Monte Carlo radiative transfer code (Pinte et al. 2006, 2009), assuming $T_{\text {gas }}=T_{\text {dust }}$, and local thermodynamic equilibrium as we are looking at low- $J$ CO lines. The central star was represented by a sphere of radius $2.1 \mathrm{R}_{\odot}$, radiating isotropically with a Kurucz spectrum at 9,250 K. We used a Voronoi tesselation where each cell corresponds to an SPH particle, avoiding the need to interpolate between the SPH and radiative transfer codes. We set the $\mathrm{CO}$ abundance following the prescription in Appendix B of Pinte et al. (2018) to account for freeze-out where $\mathrm{T}<20 \mathrm{~K}$, as well as photo-dissociation and photo-desorption in locations where the UV radiation is high. We adopted a turbulent velocity of $50 \mathrm{~m} \mathrm{~s}^{-1}$, consistent with the upper limits found by Flaherty et al. (2015) and Flaherty et al. (2017). We assumed a population of astrosilicate (Draine 2003) grains with sizes ranging from 0.03 to $1000 \mu \mathrm{m}$ and following a power-law $\mathrm{d} n(a) \propto a^{-3.5} \mathrm{~d} a$, a gas-to-dust ratio of 100 , and computed the dust optical properties using Mie theory.

Figure 5 presents the predicted emission in ${ }^{12} \mathrm{CO}$ $J=2-1$ of our theoretical models for four different planet masses. A $2 \mathrm{M}_{\text {jup }}$ planet appears to reproduce a deformation of the ${ }^{12} \mathrm{CO}$ isovelocity curve that is consistent with the observations. At $1 \mathrm{M}_{\mathrm{jup}}$, the planet only produces a small deformation that is barely visible in the channel maps, while a more massive planet triggers a strong spiral arm that would have been detected in channel maps at least up to $0.5 \mathrm{~km} \mathrm{~s}^{-1}$ from the nominal velocity of $1 \mathrm{~km} \mathrm{~s}^{-1}$. The twisted emission in the channel maps is a direct consequence of deviation from Keplerian velocity generated by the planet along the wake of the spiral arms (Fig. 4, right panel). Perez et al. (2015) also predicted that the circumplanetary disc can be detected as a compact emission separated in velocity from the circumstellar disc emission. The circumplanetary disc radius is about one-third of the Hill radius (e.g. Ayliffe \& Bate 2009). A 2-5 Jupiter mass planet would produce a circumplanetary disc with a diameter smaller than 6 $8 \mathrm{au}$, respectively. At the current spatial resolution of the ALMA observations, its flux will be diluted in the beam $(\approx 20 \mathrm{au})$.

Note that for the adopted disc parameters, the planet migrates by about 30 au during the simulation, and the synthetic maps display the velocity deviation slightly closer to the star than in the data. At this rate, the planet would reach the star in about $1 \mathrm{Myr}$ (though we overestimate the migration rate by a factor of 2-3 due to the relatively large sink particle radius that we adopted; see Ayliffe \& Bate 2010). If the detection is confirmed, the survival of such an embedded planet could put additional constraints on the disc surface density profile and viscosity.

Grady et al. (2000) detected a gap in the scattered light images with HST/STIS at $260 \mathrm{au}$, and estimated the mass of a potential planet to be $0.4 \mathrm{M}_{\text {jup }}$ (based on some simple analytical derivation). Isella et al. (2016) also detected a small dip in the integrated $\mathrm{CO}$ brightness profile at $\approx 2.2$ " (see their Figure 1 , or Figure 5 in Liu et al. 2018). In our model, the gap appears in scattered light for a planet mass larger than $2 \mathrm{M}_{\mathrm{jup}}$, but remains undetected in the synthetic CO maps. The final profile of a planetary gap establishes itself on a viscous timescale however (thousands of orbits with a viscosity of $10^{-3}$ ), however, and the gap width and depth in our models are only lower limits.

The effect of the planet appears fainter in the ${ }^{13} \mathrm{CO}$ channels maps than in the ${ }^{12} \mathrm{CO}$ maps, even if the planet is located in the midplane. This is due to optical depth and vertical temperature gradient effects: the ${ }^{12} \mathrm{CO}$ is coming from a vertically narrow and warm layer above the midplane, while the ${ }^{13} \mathrm{CO}$ is originating from a deeper, thicker layer, where the disc is almost vertically isothermal, resulting in a uniform emissivity that washes out some of the kinematics signal.

Are we seeing the signature of an embedded planet? Can we exclude wishful thinking? The strongest evidence is that the perturbation to the disc kinematics is highly localised in both space and velocity. This excludes any mechanism that merely produces axisymmetric rings in discs. This excludes, for example, ice lines (Zhang et al. 2015), self-induced dust traps (Gonzalez et al. 2015), instabilities (Takahashi \& Inutsuka 2014; Lorén-Aguilar \& Bate 2015) and zonal flows (Flock et al. 2015). A spiral wave could in principle result from the disc self-gravity, but multiple, large-scale spirals would be expected in that case (e.g. Dipierro et al. 2015) which the localized deviation seen in HD 163296 would seem to exclude.

The localised nature of the kinematic perturbation, that it occurs close to the gap found by Grady et al. (2000), and the similarity to the signatures predicted by our hydrodynamic models is a strong evidence for a young protoplanet in a gas-rich disc. However, confirmation with direct imaging is the only way to be sure. The relatively large planet mass and its known location in the disc means direct imaging follow-up might be able to detect it, depending on how embedded it is in disc. So far, no point source has so far been detected at 

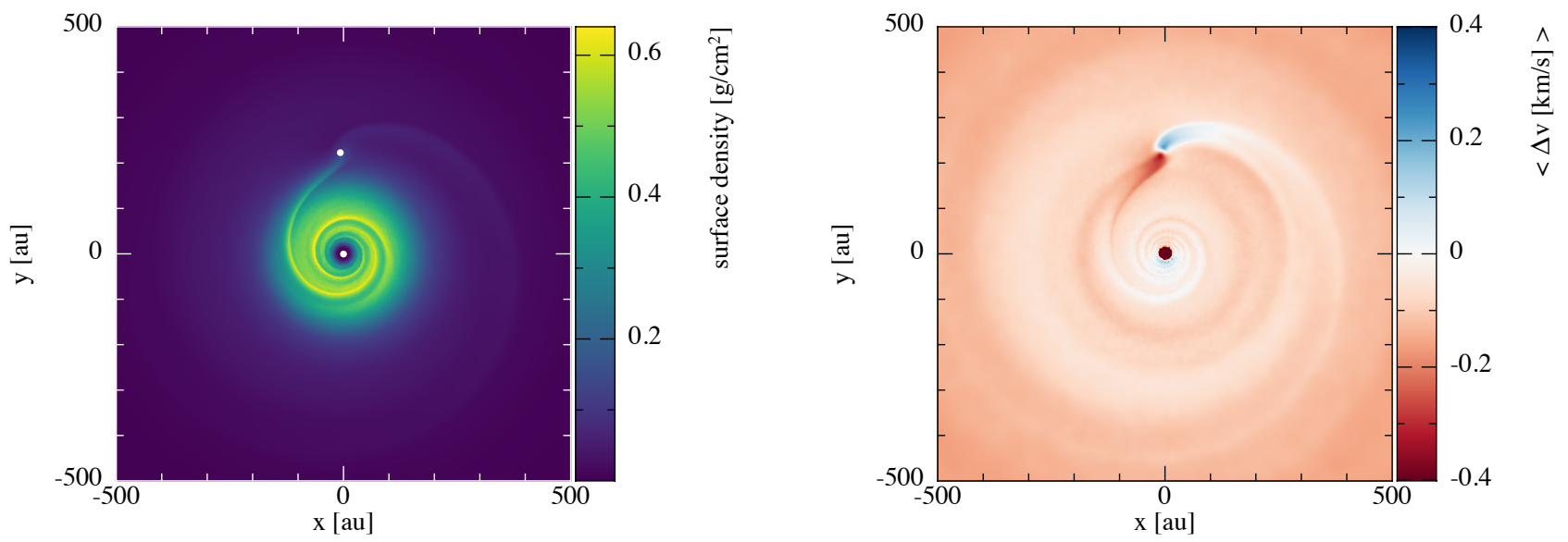

Figure 4. Left panel: surface density in 3D hydrodynamics simulations of the HD 163296 disc, shown after 35 orbits of a $2 \mathrm{M}_{\text {jup }}$ planet and viewed at a face-on inclination. Dots mark the star and planet. Right panel: deviation of the azimuthal velocity from Keplerian velocity.

the location of the potential planet with near-IR adaptive optics systems. A $2 \mathrm{M}_{\text {Jup }}$ planet is consistent with the upper limits (for an unobscured planet) obtained by adaptive optics systems, such as SPHERE (Muro-Arena et al. 2018) and Keck L' (Guidi et al. 2018). Using the formalism developed in Pinte et al. (2018), we find that the velocity kink is located at a distance of $\approx 260 \mathrm{au}$, and an elevation above the midplane of $\approx 70 \mathrm{au}$. Assuming that the potential planet is located in the midplane, it would be at a projected distance of $2.3 \pm 0.2$ " and PA $=-3 \pm 5^{\circ}$ from the star, where we estimated the uncertainties by locating the velocity deviation with half of a beam accuracy. If the planet orbit is slightly inclined compared to the disc's plane, the position on the sky will be shifted along a line going from the northeast to the southwest directions.

Can massive planets form at a distance of 250 au from the star? The location of giant planets in the outer regions of discs would be broadly consistent with gravitational instability. On the other hand, the timescale for core accretion may also be reasonable given that HD 163296 is a relatively old disc $(\approx 5 \mathrm{Myr})$. The planet may also have undergone outward migration, depending upon the initial profile of the disc. It is beyond the scope of this Letter to speculate further.

\section{SUMMARY}

We detected a $15 \%$ deviation from Keplerian flow around the star in the disc around HD 163296. The deviation was detected in both Band 6 and Band 7 in two different transitions of ${ }^{12} \mathrm{CO}$ and matches the kinematic signature predicted for an embedded protoplanet.

Comparing the observations to a series of 3D hydrodynamic and radiative transfer models of embedded plan- ets suggests that the kinematic feature is caused by a planet of of $\approx 2 \mathrm{M}_{\mathrm{Jup}}$ in the midplane. Such a planet would be located at a distance of $\approx 260$ au.

\section{ACKNOWLEDGMENTS}

This Letter makes use of the following ALMA data: ADS/JAO.ALMA\#2011.0.00010.SV and ADS/JAO.ALMA\#2013.1.00601.S. ALMA is a partnership of ESO (representing its member states), NSF (USA) and NINS (Japan), together with NRC (Canada), MOST and ASIAA (Taiwan), and KASI (Republic of Korea), in cooperation with the Republic of Chile. The Joint ALMA Observatory is operated by ESO, AUI/NRAO and NAOJ. The National Radio Astronomy Observatory is a facility of the National Science Foundation operated under cooperative agreement by Associated Universities, Inc. This work was performed on the ozSTAR national facility at Swinburne University of Technology. ozSTAR is funded by Swinburne and the Australian Government's Education Investment Fund. We thank the anonymous referee for insightful comments and suggestions. C.P. and D.J.P. acknowledge funding from the Australian Research Council via FT170100040, FT13010003,4 and DP180104235. F.M. and C.P. acknowledge funding from ANR of France (ANR-16-CE310013).

\section{Facilities: ALMA.}

Software: CASA(McMullinetal.2007), phantom(Price et al. 2017), splash (Price 2007), mcfost (Pinte et al. 2006, 2009). 


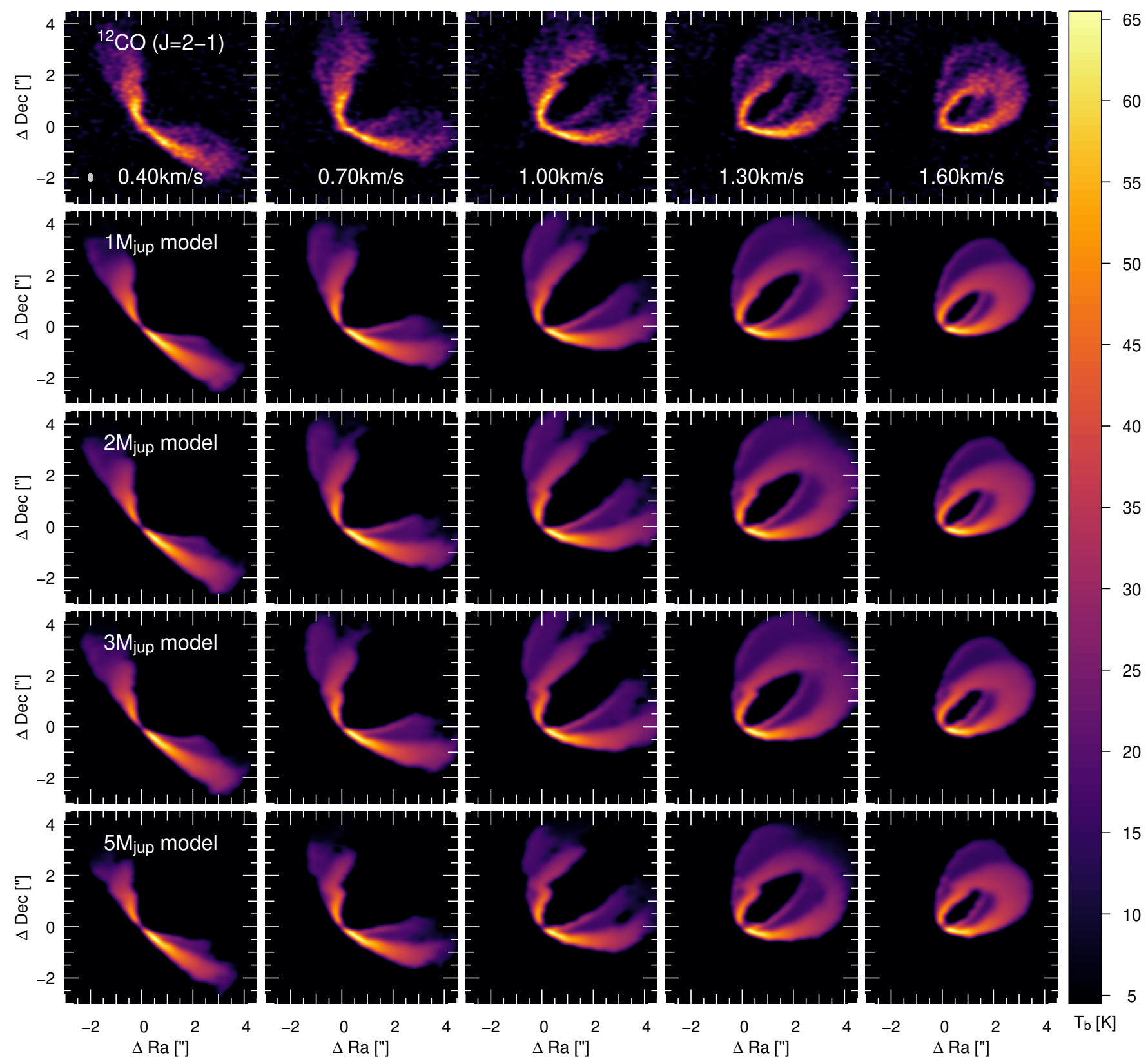

Figure 5. Comparison of ${ }^{12} \mathrm{CO} \mathrm{J}=2-1$ ALMA observations (top row) with synthetic channel maps from our 3D hydrodynamics calculations with embedded planets of $1,2,3$ and $5 \mathrm{M}_{\mathrm{Jup}}$ (from top to bottom). Channel width is $0.1 \mathrm{~km} \mathrm{~s}^{-1}$. Synthetic maps were convolved to a Gaussian beam to match the spatial resolution of the observations .

\section{REFERENCES}

ALMA Partnership, Brogan, C. L., Pérez, L. M., et al. 2015, ApJL, 808, L3, doi: 10.1088/2041-8205/808/1/L3

Andrews, S. M., Wilner, D. J., Zhu, Z., et al. 2016, ApJL, 820, L40, doi: 10.3847/2041-8205/820/2/L40

Ayliffe, B. A., \& Bate, M. R. 2009, MNRAS, 397, 657,

doi: 10.1111/j.1365-2966.2009.15002.x

-. 2010, MNRAS, 408, 876,

doi: 10.1111/j.1365-2966.2010.17221.x
Bate, M. R., Bonnell, I. A., \& Price, N. M. 1995, MNRAS, 277,362

Benisty, M., Juhasz, A., Boccaletti, A., et al. 2015, A\&A, 578, L6

Béthune, W., Lesur, G., \& Ferreira, J. 2016, A\&A, 589, A87 Biller, B. A., Males, J., Rodigas, T., et al. 2014, ApJL, 792, L22, doi: 10.1088/2041-8205/792/1/L22 
Brittain, S. D., Carr, J. S., Najita, J. R., Quanz, S. P., \& Meyer, M. R. 2014, ApJ, 791, 136, doi: 10.1088/0004-637X/791/2/136

Casassus, S., Marino, S., Pérez, S., et al. 2015, ApJ, 811, 92

Currie, T., Cloutier, R., Brittain, S., et al. 2015, ApJL, 814, L27, doi: 10.1088/2041-8205/814/2/L27

de Gregorio-Monsalvo, I., Ménard, F., Dent, W., et al. 2013, A\&A, 557, A133

Dipierro, G., Pinilla, P., Lodato, G., \& Testi, L. 2015, MNRAS, 451, 974, doi: 10.1093/mnras/stv970

Draine, B. T. 2003, ApJ, 598, 1017, doi: 10.1086/379118

Flaherty, K. M., Hughes, A. M., Rosenfeld, K. A., et al. 2015, ApJ, 813, 99

Flaherty, K. M., Hughes, A. M., Rose, S. C., et al. 2017, ApJ, 843, 150

Flock, M., Ruge, J. P., Dzyurkevich, N., et al. 2015, A\&A, 574, A68, doi: 10.1051/0004-6361/201424693

Gaia Collaboration, Brown, A. G. A., Vallenari, A., et al. 2018, ArXiv e-prints. https://arxiv.org/abs/1804.09365

Gonzalez, J.-F., Laibe, G., Maddison, S. T., Pinte, C., \& Ménard, F. 2015, MNRAS, 454, L36, doi: $10.1093 / \mathrm{mnrasl} / \mathrm{slv} 120$

Grady, C. A., Devine, D., Woodgate, B., et al. 2000, ApJ, 544,895

Guidi, G. et al. 2018, submitted

Isella, A., Guidi, G., Testi, L., et al. 2016, Physical Review Letters, 117, 251101

Kraus, A. L., \& Ireland, M. J. 2012, ApJ, 745, 5, doi: 10.1088/0004-637X/745/1/5

Ligi, R., Vigan, A., Gratton, R., et al. 2018, MNRAS, 473, 1774, doi: 10.1093/mnras/stx2318

Liu, S.-F., Jin, S., Li, S., Isella, A., \& Li, H. 2018, ArXiv e-prints. https://arxiv.org/abs/1803.05437

Lodato, G., \& Price, D. J. 2010, MNRAS, 405, 1212

Lorén-Aguilar, P., \& Bate, M. R. 2015, MNRAS, 453, L78, doi: 10.1093/mnrasl/slv109

McMullin, J. P., Waters, B., Schiebel, D., Young, W., \& Golap, K. 2007, in Astronomical Society of the Pacific Conference Series, Vol. 376, Astronomical Data Analysis Software and Systems XVI, ed. R. A. Shaw, F. Hill, \& D. J. Bell, 127

Muro-Arena, G. A., Dominik, C., Waters, L. B. F. M., et al. 2018, ArXiv e-prints. https://arxiv.org/abs/1802.03328
Natta, A., Testi, L., Neri, R., Shepherd, D. S., \& Wilner, D. J. 2004, A\&A, 416, 179, doi: 10.1051/0004-6361:20035620

Ogilvie, G. I., \& Lubow, S. H. 2002, MNRAS, 330, 950, doi: 10.1046/j.1365-8711.2002.05148.x

Perez, S., Dunhill, A., Casassus, S., et al. 2015, ApJL, 811, L5

Pinte, C., Harries, T. J., Min, M., et al. 2009, A\&A, 498, 967, doi: 10.1051/0004-6361/200811555

Pinte, C., Ménard, F., Duchêne, G., \& Bastien, P. 2006, A\&A, 459, 797, doi: 10.1051/0004-6361:20053275

Pinte, C., Ménard, F., Duchêne, G., et al. 2018, A\&A, 609, A 47

Price, D. J. 2007, PASA, 24, 159

Price, D. J., Wurster, J., Nixon, C., et al. 2017, ArXiv e-prints. https://arxiv.org/abs/1702.03930

Price, D. J., Cuello, N., Pinte, C., et al. 2018, MNRAS, doi: $10.1093 / \mathrm{mnras} / \mathrm{sty} 647$

Quanz, S. P., Amara, A., Meyer, M. R., et al. 2015, ApJ, 807, 64, doi: 10.1088/0004-637X/807/1/64

—. 2013a, ApJL, 766, L1, doi: 10.1088/2041-8205/766/1/L1

Quanz, S. P., Avenhaus, H., Buenzli, E., et al. 2013b, ApJL, 766, L2, doi: 10.1088/2041-8205/766/1/L2

Rameau, J., Follette, K. B., Pueyo, L., et al. 2017, AJ, 153, 244, doi: 10.3847/1538-3881/aa6cae

Reggiani, M., Quanz, S. P., Meyer, M. R., et al. 2014, ApJL, 792, L23, doi: 10.1088/2041-8205/792/1/L23

Reggiani, M., Christiaens, V., Absil, O., et al. 2018, A\&A, 611, A74, doi: 10.1051/0004-6361/201732016

Rosenfeld, K. A., Andrews, S. M., Hughes, A. M., Wilner, D. J., \& Qi, C. 2013, ApJ, 774, 16

Sallum, S., Follette, K. B., Eisner, J. A., et al. 2015, Nature, 527, 342, doi: 10.1038/nature15761

Shakura, N. I., \& Sunyaev, R. A. 1973, A\&A, 24, 337

Stolker, T., Dominik, C., Avenhaus, H., et al. 2016, ArXiv e-prints

Takahashi, S. Z., \& Inutsuka, S.-i. 2014, ApJ, 794, 55, doi: 10.1088/0004-637X/794/1/55

Thalmann, C., Mulders, G. D., Janson, M., et al. 2015, ApJL, 808, L41, doi: 10.1088/2041-8205/808/2/L41

Thalmann, C., Janson, M., Garufi, A., et al. 2016, ApJL, 828, L17, doi: 10.3847/2041-8205/828/2/L17

Walsh, C., Daley, C., Facchini, S., \& Juhász, A. 2017, A\&A, 607, A114, doi: 10.1051/0004-6361/201731334

Weaver, E., Isella, A., \& Boehler, Y. 2018, ApJ, 853, 113, doi: 10.3847/1538-4357/aaa481

Zhang, K., Blake, G. A., \& Bergin, E. A. 2015, ApJL, 806, L7, doi: 10.1088/2041-8205/806/1/L7 